\title{
Improvement of Traffic Network Reliability after a Disaster Based on Local Government Support
}

\author{
Shuming FANG* \\ Hiroshi WAKABAYASHI $* *$ \\ *Doctoral course student, Graduate School of the Faculty of Urban Science, Meijo University \\ **Professor, Faculty of Urban Science, Meijo University
}

(Received October 2, 2012 Accepted November 5, 2013)

\begin{abstract}
Highly reliable traffic networks on highways and roadways are very important during abnormal periods, such as during disasters. Network reliability can be improved effectively by focusing on the most important key link in the network. Although many indices have been proposed for identifying the most important key link, numerous shortcomings associated with these indices make it difficult to obtain a good solution for evaluating network reliability improvement. In addition, an investment strategy based on local government support is also important for improving network reliability. However, the capital available from local government for traffic restoration is usually limited after a disaster. This paper therefore proposes an optimal method of network reliability improvement based on local government support combined with limited capital. To determine the efficiency of the model for network reliability improvement, simulations for a simple parallel network and a simple bridge network are conducted. A general algorithm for identifying the most important key link in any network is then developed based on these simulations. Lastly, a rational conclusion for network reliability improvement is presented.
\end{abstract}

Keyword: Network reliability, Reliability increase, Local government support, Importance index, Disaster.

\section{Introduction}

It is important to maintain a highly reliable traffic network of highways and roadways for abnormal periods, especially during a disaster. However, the traffic systems of disaster areas may be seriously damaged, and deciding which damaged road should be improved first to maintain or improve traffic network reliability is very difficult. In general, network reliability can be greatly improved by improving the key link in the network; the most important key link is the link to improve network reliability to the great- est degree. Thus, indices such as reliability importance $(R I)$ (Barlow et al., 1975) and criticality importance (CI) (Henley et al., 1981) have been proposed. However, these indices have their own shortcomings for finding the most important key link in some types of networks. In addition, at the first stage of a disaster, the cost of repairing the damaged traffic system is important for improving traffic network reliability.

In China, local government is responsible for reconstruction after a disaster, even though the government most likely has limited capital. Therefore, maximizing traffic network reliability as soon as possible 
primarily depends on local government support after a disaster in China. In practice, the decisions of local government in selecting the most important key link are affected by many factors, such as the population of the disaster area near a damaged road, the damage level of other roads, the economic status of industrial areas near a damaged main road, and so on. For example, the Chinese government first allocates the most resources to repair damaged roads in disaster regions that are considered the most essential for strategic or economic reasons.

However, local government support was first overlooked in current indices such as probability importance and criticality importance. Nevertheless, to improve network reliability after a disaster, the effect of local government support on finding the most important key link should be taken seriously and discussed carefully.

\section{Current Importance Indices}

The concept of importance indices has long been proposed in the field of systems engineering, but has appeared in only a few papers in the transportation field (Barlow, 1969). Importance is defined as the degree of magnitude to which an improvement in link reliability contributes to system reliability. The indices of importance proposed in this paper are based on connectivity reliability (also referred to as terminal reliability).

\subsection{Terminal Reliability}

The terminal reliability of a highway network is defined as the probability that two given nodes over the network are connected with a certain service level of traffic for a given time period (Iida and Wakabayashi, 1989; Wakabayashi and Iida, 1992). Similarly, link reliability in the network is defined as the probability that the traffic reaches a certain service level for a given time period. Terminal reliability, $R$, is given by an expression using minimal-path sets, as follows (Iida and Wakabayashi, 1990):

$$
R(r)=E\left[1-\prod_{S=1}^{p}\left(1-\prod_{a \in P_{s}} X_{a}\right)\right]
$$

where $P_{S}$ is the $S$ th minimal-path set and $p$ is the total number of minimal-path sets. This calculation method is based on the Boolean absorption method (Waka- bayashi and Iida, 1992). Here, $X_{a}$ is the binary indicator variable for link $a$ :

$$
X_{a}=\left\{\begin{array}{l}
1, \text { if link } a \text { provides a certain service level } \\
0, \text { otherwise }
\end{array}\right.
$$

Link reliability, $r_{a}$, is defined as

$r_{a}=E\left[X_{a}\right]$.

The terminal reliability of a traffic network depends on the network structure and link reliabilities. Therefore, two basic approaches have been taken to improve network reliability: to improve the network structure or to improve the reliability of the links. The focus here is on identifying which links should be improved to maximize the improvement in network reliability.

\subsection{Birnbaum Structural Importance}

(1) Definition of Birnbaum structural importance

To find the key link for improving terminal reliability efficiently, the Birnbaum structural importance index was proposed (Birnbaum, 1969). Birnbaum structural importance is defined as

$$
P I_{a}=\frac{\partial R(r)}{\partial r_{a}}, 0 \leq P I_{a} \leq 1 .
$$

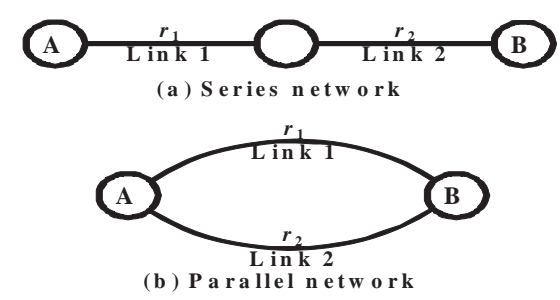

Fig. 1 Simple series and parallel networks

Birnbaum structural importance indicates the impact of a link, such that an increase or decrease in the reliability of the link affects increase or decrease in terminal reliability. Birnbaum structural importance is also known as reliability importance.

\section{(2) Advantages and shortcomings of Birnbaum structural importance}

Although Birnbaum structural importance has the potential to improve network reliability, it has a disadvantage, which is discussed in this section.

For the case of two links in a series network as shown in Fig. 1.a, terminal reliability $R_{A B}$ is shown in Eq. (5): 


$$
R_{A B}=r_{1} r_{2} \text {. }
$$

Here, $r_{1}$ and $r_{2}$ are the reliabilities for link 1 and link 2 , respectively.

For the case of two links in a parallel network as shown in Fig. 1.b, terminal reliability $R_{A B}$ is shown in Eq. (6):

$$
R_{A B}=1-\left(1-r_{1}\right)\left(1-r_{2}\right) \text {. }
$$

The two values of Birnbaum structural importance for a series network, $R I_{1}$ and $R I_{2}$, are obtained from Eq. (4) and Eq. (5) as

$$
\begin{aligned}
& R I_{1}=r_{2} \text { and } R I_{2}=r_{1} . \\
& \text { It follows that } \\
& R I_{1}>R I_{2} \text {, if } r_{1}<r_{2} .
\end{aligned}
$$

Eq. (8) indicates that improving the least reliable link in a series-type network is most effective for improving terminal reliability. This fact is easily expanded for large series-type networks. This result for improving, managing, and reconstructing a network is the expected result.

$R I_{1}$ and $R I_{2}$ for these two links in a parallel network are obtained from Eq. (4) and (6) as

$$
R I_{1}=1-r_{2} \text { and } R I_{2}=1-r_{1} \text {. }
$$

It follows that

$$
R I_{1}<R I_{2} \text {, if } r_{1}<r_{2} \text {. }
$$

The result from Eq. (10) indicates that improving the most reliable link in a parallel-type network is more effective for improving terminal reliability. Usually, however, it is difficult to improve a more reliable link, whereas it is rather easy to improve a less reliable link (Barlow and Proschan, 1975). This result is counter to what one would expect for improving, managing, and reconstructing a network.

\subsection{Criticality Importance}

\section{(1) Definition of criticality importance}

Because of the shortcoming of Birnbaum structural importance, the criticality importance index $(C I)$ was proposed. CI is the ratio of the proportional improvement in network reliability to the proportional improvement in link reliability (Henley and Kumamoto, 1992):

$$
C I_{a}=\frac{\partial R / R}{\partial r_{a} / r_{a}}=P I_{a} \frac{r_{a}}{R} .
$$

\section{(2) Advantages and shortcomings of criticality importance}

Criticality importance also has a shortcoming, which is discussed in this section.

For the case of two links in a series network, it follows from Eq. (4), (5), (7), and (11) that

$$
C I_{1}=\frac{r_{1} r_{2}}{R}=C I_{2} \text {. }
$$

This result suggests that the criticality importance index is the same for both links in a series network. However, in a series network, it is reasonable to strengthen a less reliable link, and this is thus a shortcoming of the criticality importance index. In addition, it does not provide information to distinguish between two links in terms of improving network reliability.

For the case of two links in a parallel network, it follows from Eq. (4), (6), (9), and (11) that

$$
C I_{1}=\frac{r_{1}-r_{1} r_{2}}{R}
$$

and

$$
C I_{2}=\frac{r_{2}-r_{1} r_{2}}{R}
$$

It follows that

$$
C I_{1}<C I_{2} \text {, if } r_{1}<r_{2} \text {. }
$$

Therefore, the criticality importance index also indicates that in the case of a parallel-type network, improving a more reliable link further increases the terminal reliability of the network. The results for a parallel network provided by both $R I$ and $C I$ suggest that a less reliable link should be ignored in a parallel system. In other words, people who live along a less reliable link would be neglected after a disaster. This is not reasonable planning for disaster prevention and reduction. Thus, this result is not expected.

\subsection{Advanced Criticality Importance \\ (1) Definition of advanced criticality importance proposed by Wakabayashi}

Based on the shortcomings of Eq. (10), (12), and (15), Birnbaum structural importance and criticality importance do not reflect the fact that it is more difficult to improve a more reliable link than to improve a less reliable link. Thus, it is convenient to define importance as the proportion of the marginal change in terminal reliability against the marginal change in link reliability. Changing the definition of the equation in 
reliability engineering, the criticality importance index proposed by Wakabayashi (CIW) is defined as Eq. (16) (Wakabayashi, 2004):

$$
C I W_{a}=\lim _{\Delta q_{a} \rightarrow 0}\left\{-\frac{\Delta R(r) / R(r)}{\Delta q_{a} / q_{a}}\right\}=P I_{a} \frac{1-r_{a}}{R},
$$

where $q_{a}=1-r_{a}$ is the unreliability of link $a$.

(2) Advantages and shortcomings of advanced criticality importance

For the case of two links in a series network, it follows from Eq. (4), (5), (7), and (16) that

$$
C I W_{1}=\frac{1-r_{1}}{r_{1}}
$$

and

$$
C I W_{2}=\frac{1-r_{2}}{r_{2}} \text {. }
$$

It also follows that

$C I W_{1}>C I W_{2}$, if $r_{1}<r_{2}$.

Thus, in a series-type network, advanced criticality importance has the same property as Birnbaum structural importance, and this property from Eq. (19) is exactly as one would expect.

For the case of two links in a parallel network, it follows from Eq. (4), (6), (9), and (16) that

$$
C I W_{1}=\frac{\left(1-r_{1}\right)\left(1-r_{2}\right)}{r_{1}+r_{2}-r_{1} r_{2}}=C I W_{2} .
$$

From Eq. (20), although the advanced criticality importance index is better than that proposed by Henley and Kumamoto (1981), this index is the same for both links in a parallel network, so it does not provide information to distinguish between them in terms of improving network reliability.

The indices RI, CI, and CIW discussed above, because of their own shortcomings, cannot be used to select the most important key link of a traffic network. Therefore, a good solution cannot be obtained by these indices for evaluating the improvement of network reliability. In addition, although the cost-benefit ratio is also important (Nicholson, 2007), these indices cannot predict the increase in cost for improving link reliability when link reliability increases. Thus, traffic network reliability increase in accordance with a different investment strategy by local government should be discussed.

\section{Model of Improvement of Traffic Network Reliability Based on Local Government Support}

As described in Chapter 2, a different investment strategy by local government is useful for selecting the most important key link. In fact, only limited capital may be available for improving link reliability at the first stage of a disaster. In general, different local governments use different investment strategies and provide different support to improve network reliability in China. Thus, the effect of local government support on repairing damaged traffic systems should be discussed based on limited capital.

\subsection{Local Government Support}

In China, local government plays an important role in reconstructing a damaged traffic network and maintains traffic order after a disaster. Not only is the limited capital appropriated from the Ministry of Finance and that from the local finance department distributed to disaster areas by local government, but all kinds of nonmaterial support such as volunteers, psychological counseling for victims, and so on are also arranged by local government. In other words, local government can provide material support and nonmaterial support. In general, material support is usually considered to be money or goods, and nonmaterial support includes many aspects such as civil technology support, volunteers for reconstructing the traffic system or maintaining traffic order, and so forth.

In addition, a pairing support policy is being implemented in China. Pairing support is a system to support disaster areas by activating nationwide help. Other local governments of non-disaster areas provide special partnership assistance support, especially nonmaterial support, to the local government of a disaster area when a disaster happens.

\subsection{Assumptions before Modeling}

Some assumptions are given so as to discuss the efficiency of local government support in maximizing improvement of network reliability in the case of limited capital:

1) The cost of making the link reliability of link $a$ reach 1.0 tends to infinity when local gov ernment does not provide any support for link $a$.

2) The maximum capital for improving network reliability is limited. 
3) Although reliability equals zero, the original cost does not equal zero because of the basic work for increasing the link reliability.

4) The support provided by local government for a certain link is limited.

5) The support provided by local government for a certain link is only nonmaterial support because nonmaterial support is more varied than other factors at the first stage of a disaster.

\subsection{Modeling}

Based on the abovementioned assumptions, the relationship between the increase in cost and the increase in link reliability can be formulated.

If the available capital for a link with reliability $r_{a}$ is $C_{a}$, the cost of improving link reliability increases as link reliability increases by assuming the proportion of marginal capital $d C_{a}$ against marginal link reliability increase $d r_{a}$, and the relationship between $d C_{a}$ and $d r_{a}$ is shown as follows:

$$
\frac{d C_{a}}{d r_{a}}=\alpha e^{\frac{r_{a}}{\beta_{a}}} .
$$

$\alpha$ : Positive constant, where $d C_{a} / d r_{a}$ equals $\alpha$ when $r_{a}$ equals zero.

$\beta_{a}$ : Non-negative constant that is local government support for link $a$, where $\beta_{a}$ equals zero when local government cannot provide any support, and $d C_{a} / d r_{a}$ tends to infinity.

Based on Eq. (21), the cost-reliability function is shown as Eq. (22):

$$
C_{a}=\alpha \beta_{a} e^{\frac{r_{a}}{\beta_{a}}}+C_{0}
$$

Because of assumption 3), $C_{0}$ is the value of $\left(C_{a}-\alpha \beta_{a}\right)$ when $r_{a}$ equals zero. When the link reliability of link $a$ increases from $r_{a}$ to $r_{a}+\Delta r_{a}$, the required capital is calculated as

$$
\delta C_{a}=\alpha \beta_{a} e^{\frac{r_{a}+\Delta r_{a}}{\beta_{a}}}-\alpha \beta_{a} e^{\frac{r_{a}}{\beta_{a}}}
$$

If $N$ links are in the traffic network of a disaster area, the total capital for improving network reliability is shown in Eq. (24):

$$
C_{\text {increase }}=\sum_{a=1}^{N} \delta C_{a} .
$$

At the first stage of a disaster, the capital for im- proving network reliability is invariably limited; thus, maximizing network reliability increase based on limited capital becomes a nonlinear optimization problem, shown as the following:

$$
\begin{array}{ll}
\text { Maximize: } & \not R=R-R_{0} ; \\
\text { Subject to: } & r_{i 0} \leq r_{i} \leq 1, i \in(1, \eta \eta) \\
& C_{\text {increase }}=C ; \\
& \beta_{i} \leq B ; \\
& r_{i 0}=D(D \leq 1) .
\end{array}
$$

$B$ : An exogenous variable that reflects entire local government support in the damaged traffic network;

$C$ : A constant that means the limited capital for traffic restoration;

D: A constant;

$n$ : Number of links in the whole network;

$r_{i 0}$ : Original reliability of link $i$;

$R$ : Current traffic network reliability;

$R_{0}$ : Original traffic network reliability;

$\delta R$ : Network reliability increase.

\section{Simulation of the Model}

In this chapter, to find the most important key link, the most optimized improvement of the whole network reliability as a standard nonlinear optimization problem is simulated by using the mathematic software LINGO for a simple parallel network and a simple bridge network.

\subsection{Effect of Government Support on a Simple Parallel Network}

To find the most important key link, the following three cases show the difference in the original link reliability between two links in a parallel network based on different levels of government support between two links: a very small difference $\left(r_{10}=0.45\right.$ and $\left.r_{20}=0.55\right)$, a great difference $\left(r_{10}=0.3\right.$ and $\left.r_{20}=0.7\right)$, and a very great difference $\left(r_{10}=0.1\right.$ and $\left.r_{20}=0.9\right)$.

\section{(1) A very small difference in the original link reliability between two links}

Figure 2 includes four cases that show improvement of link reliability and network reliability based on local government support for two links when $r_{10}=$ 0.45 and $r_{20}=0.55$. The primary horizontal axis of every case in Fig. 2 is local government support for link 1 , the secondary horizontal axis is local government 


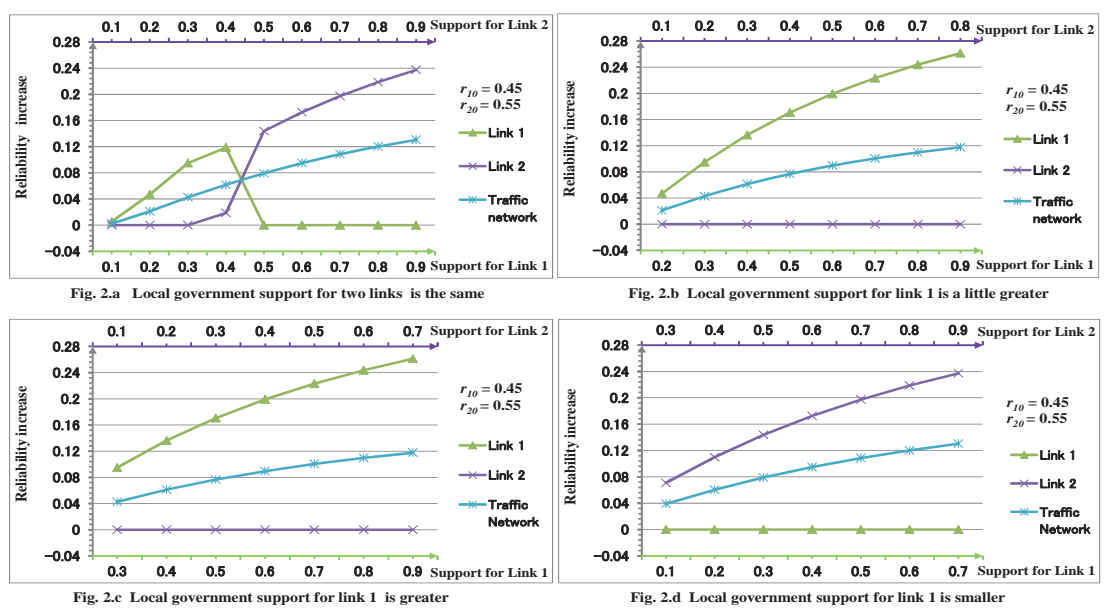

Fig. 2 Results for a very small difference in original link reliability between two links

support for link 2, and the primary vertical axis is the reliability increase of the links and traffic network. As shown in Fig. 2.a, if local government support for two links is the same and relatively small, a less reliable link should be selected as the most important key link. On the other hand, a more reliable link should be selected if local government support for two links is the same but relatively great as shown in Fig. 2.a. From Fig. 2.b and Fig. 2.c, when local government support for two links is different, the link providing more local government support should be improved first and the other link should be ignored.

\section{(2) A great difference in original link reliability between two links}

Figure 3 shows the reliability increase based on local government support for two links when $r_{10}=0.3$ and $r_{20}=0.7$. Link 1 should be selected as the most important key link if it satisfies one of the following conditions:
1) Local government support for link 1 is much greater than that for link 2 as shown in Fig. 3.d;

2) Local government support for two links is relatively small as shown in Fig. 3.a, Fig. 3.b, Fig. 3.c and Fig. 3.e when local government support for link 2 is not greater than that for link 1 .

Link 2 should be selected as the most important key link when the above two conditions cannot be satisfied.

Any link can be selected to improve at the point at which the curve of link 1 crosses that of link 2 in Fig. 3.a c.

\section{(3) A very great difference in original link reliability between two links}

Figure 4 shows the reliability increase based on local government support for two links when $r_{10}=0.1$ and $r_{20}=0.9$. Link 1 should be selected as the most important key link only when local government support for the two links is relatively small and local
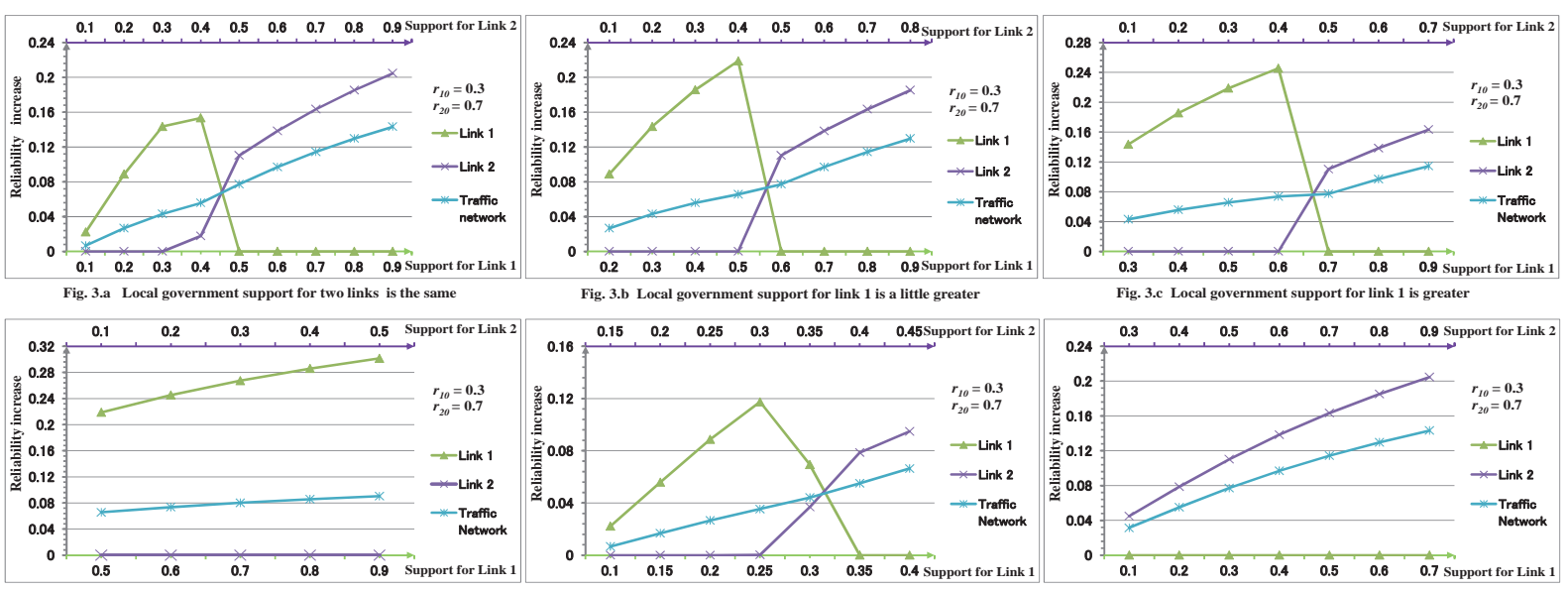

Fig. 3 Results for a great difference in original link reliability between two links 

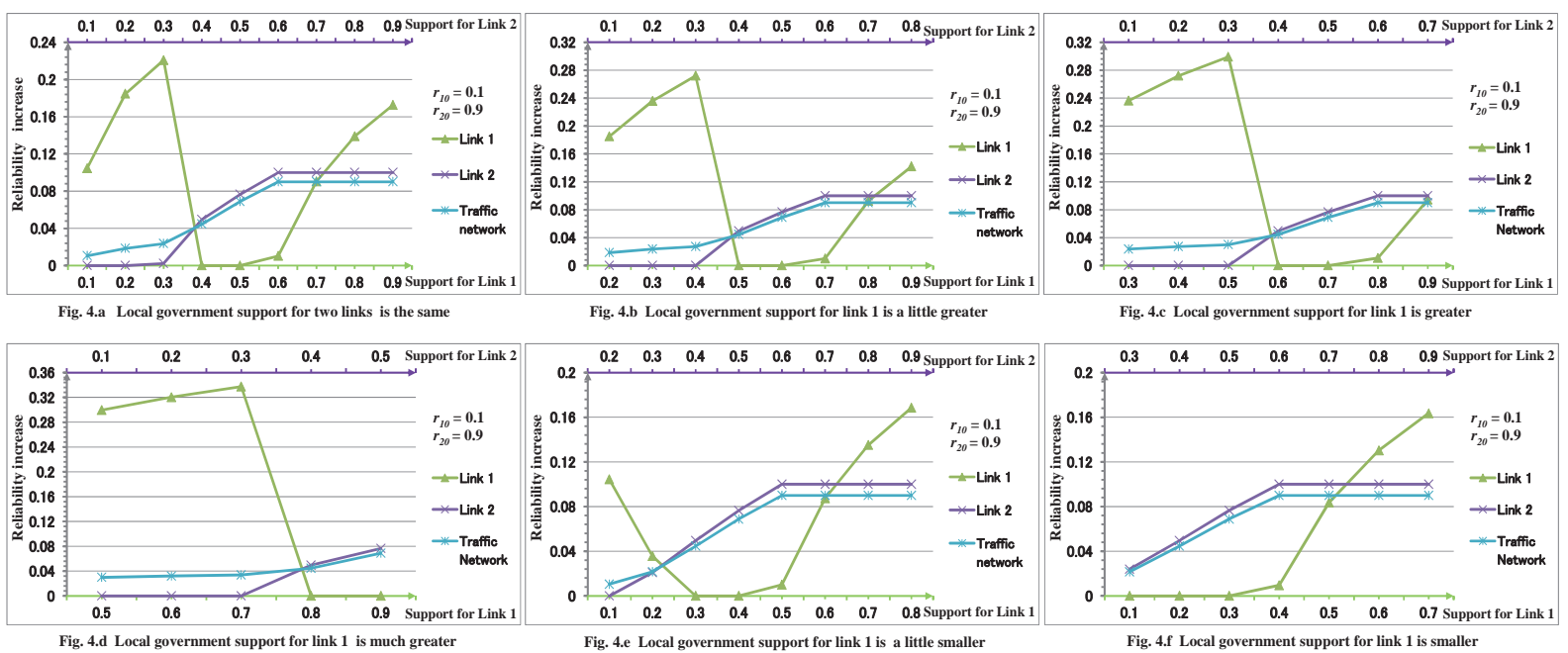

Fig. 4 Results for a very great difference in original link reliability between two links

government support for link 2 is not greater than local government support for link 1 as shown in Fig. 4.a e.

On the other hand, link 2 should be selected and link 1 should be ignored when local government support for the two links is relatively great as shown in Fig. 4.a e. or when government support for link 2 is greater than that for link 1 as shown in Fig. 4.f. A special point for attention is that the reliability of link 1 begins to increase when the reliability of link 2 becomes 1.0 as shown in Fig. 4.e and Fig. 4.f ( $r_{20}=0.9$; the reliability increase of link 2 reaches 0.1 ).

Obviously, after the reliability of a higher-reliability link ( $\left.r_{20}=0.9\right)$ becomes 1.0 , the lower-reliability link will be improved no matter to what extent it receives local government support as shown in Fig. 4. In other words, there must be a positive reliability increase of link 1 if that of link 2 reaches 0.1 .

In general, local government support for different disaster areas will not be considerably different. Therefore, link 1 as a less reliable link should be selected

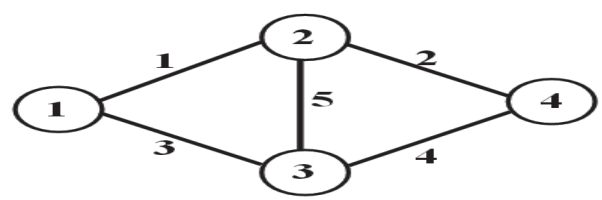

Fig. 5 A simple bridge network

as the most important key link when government support for two links is relatively small as in the abovementioned three cases. On the other hand, a more reliable link should be selected when local gov- ernment support is relatively great as shown in Fig. 3.a c, Fig. 3.e, Fig. 4.a c, and Fig. 4.e.

\subsection{Effect of Government Support on a Simple Bridge Network}

As shown in Fig. 5, a simple bridge network is used here. The network has four nodes and five links. The minimal-path sets of this bridge are shown as: $P_{1}=\{1,2\}, P_{2}=\{3,4\}, P_{3}=\{1,5,4\}$, and $P_{4}=\{3,5,2\}$ where $P_{1}$ and $P_{2}$ are the primary minimal-path sets (Iida, Y. et al., 1988, 1990) (Wakabayashi, H. et al., 1991, 1992).

Since the independent path set is a set of links in a series system, the reliability of one path set is a combination of link reliability (Wakabayashi et al., 1992). When the most important key path set is found, the most important key link belonging to the most important key path set can be found according to $R I$ and $C I W$. The reliability of every path set is shown in the following:

$$
R\left(P_{1}\right)=r_{1} r_{2}, R\left(P_{2}\right)=r_{3} r_{4}, R\left(P_{3}\right)=r_{1} r_{5} r_{4}, R\left(P_{4}\right)=r_{3} r_{5} r_{2} .
$$

The exact value of network reliability for the bridge network is shown in Eq. (27) by using Boolean absorption (Wakabayashi, H. et al., 1992).

$$
\begin{array}{r}
R(r)=r_{1} r_{2}+r_{3} r_{4}+r_{1} r_{5} r_{4}+r_{3} r_{5} r_{2}-r_{1} r_{2} r_{3} r_{4}-r_{1} r_{2} r_{4} r_{5} \\
-r_{1} r_{3} r_{4} r_{5}-r_{1} r_{2} r_{3} r_{5}-r_{2} r_{3} r_{4} r_{5}+2 r_{1} r_{2} r_{3} r_{4} r_{5} .
\end{array}
$$

To simplify the simulation, only the original reliability of link 1 and link 3 varies, and the original reliability of the other links is fixed as 0.5 .

Three cases of difference in original reliability 
between link 1 and link 3 are presented based on local government support for all links, and the three cases are as follows: a small difference $\left(r_{10}=0.4\right.$ and $r_{30}=$ $0.6)$, a great difference $\left(r_{10}=0.3\right.$ and $\left.r_{30}=0.7\right)$, and a very great difference $\left(r_{10}=0.1\right.$ and $\left.r_{30}=0.9\right)$. The original reliability of every minimal-path set is different as $R\left(P_{1}\right)>R\left(P_{2}\right)>R\left(P_{3}\right)>R\left(P_{4}\right)$ in all cases.

\section{(1) Case 1: A small difference in original link reliability between link 1 and link 3}

Figure 6 shows the reliability increase based on the level of local government support for all links when $r_{10}=0.4$ and $r_{30}=0.6$. The primary horizontal axis of every case in Fig. 6 is local government support for link 1 or link 3 , the secondary horizontal axis is local government support for other links, and the primary vertical axis is the reliability increase of every minimal-path set and the traffic network.

From Fig. 6, if local government support for all links is the same and relatively small, a less reliable primary minimal-path set, $P_{1}$, should be selected as the most important key path set, and link 1 of $P_{1}$ is the most important key link. On the other hand, a more reliable primary minimal-path set, $P_{2}$, should be selected if local government support for all links is the same and relatively great, and link 4 of $P_{2}$ should be selected as the most important key link.

When local government support for link 1 and link 3 is different, minimal-path set $P_{1}$ should be selected as the most important key path set by satisfying one of the following conditions:

1) Local government support for the links of $P_{1}$ is much greater than that for the links of $P_{2}$;
2) Local government support for all links is relatively small and local government support for the links of $P_{2}$ is smaller than local government support for the links of $P_{1}$.

$P_{2}$ should be selected as the most important key path set when the above two conditions cannot be satisfied.

\section{(2) Case 2: A great difference in original link reliability between link 1 and link 3}

Figure 7 shows the reliability increase based on local government support for all links when $r_{10}=0.3$ and $r_{30}=0.7$ and the reliability of other links is 0.5 .

In this case, if local government support for all links is the same, the results for finding the most important key link are same as those for Case 1 .

When local government support for link 1 and link 3 is different, $P_{2}$ should be selected by satisfying one of the following conditions:

1) Local government support for the links of $P_{2}$ is greater than that for the links of $P_{1}$;

2) Local government support for the links is relatively great and local government support for the links of $P_{1}$ is not much greater than local government support for the links of $P_{2}$.

$P_{1}$ should be selected as the most important key path set when the above two conditions cannot be satisfied.

\section{(3) Case 3: A great difference in original link reliability between link 1 and link 3}

Figure 8 shows the reliability increase based on the variety of local government support for all links when $r_{10}=0.1$ and $r_{30}=0.9$ and the reliability of other
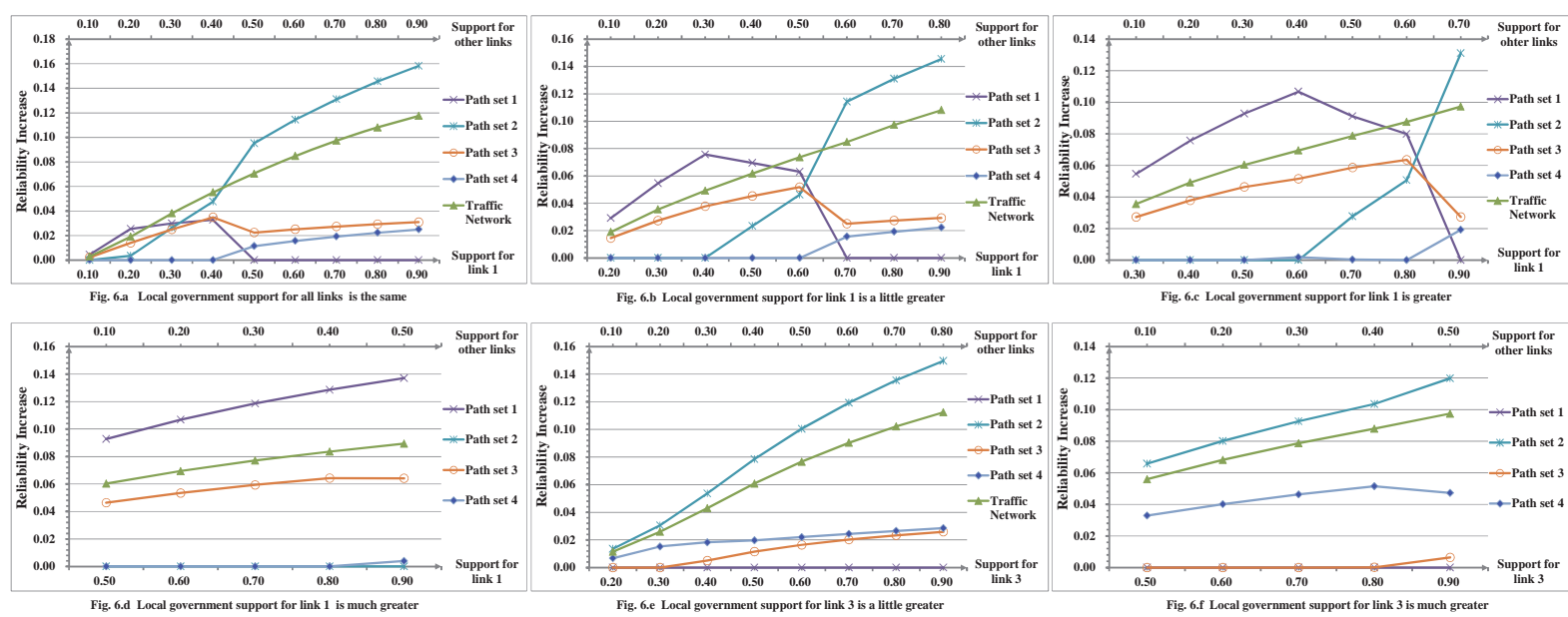

Fig. 6 Results for a small difference in original link reliability between link 1 and link 3 

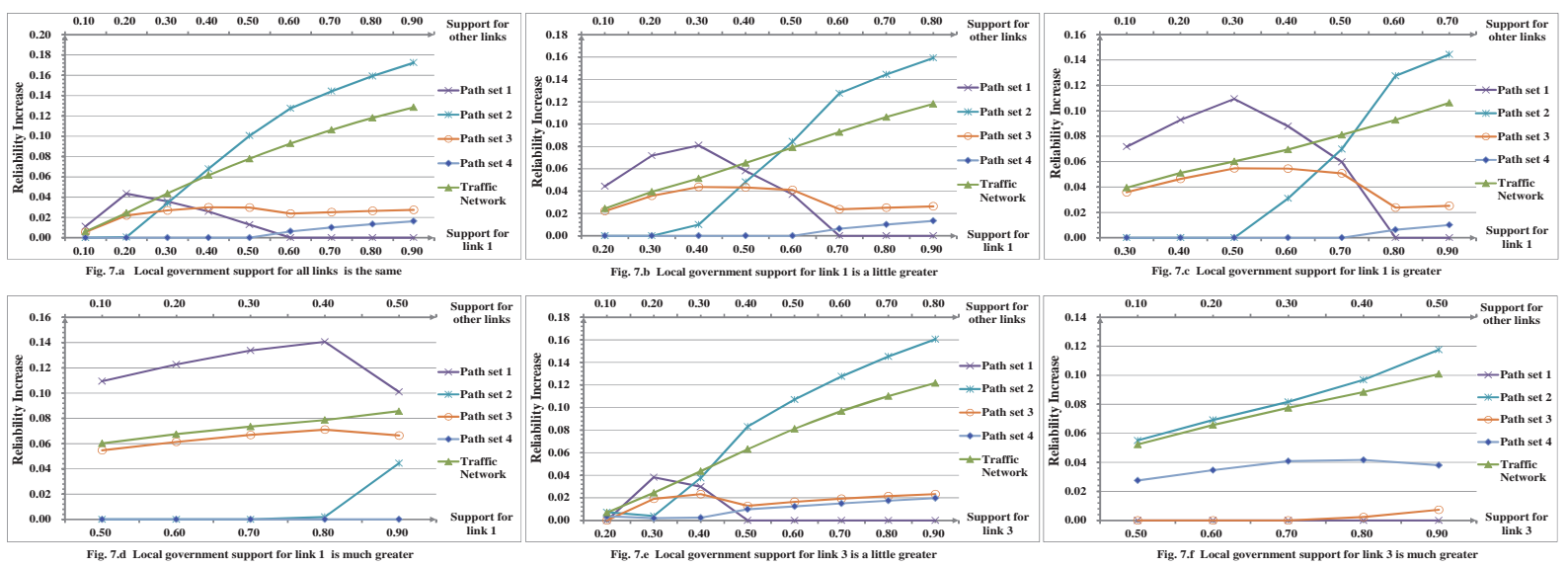

Fig. 7 Results for a great difference in original link reliability between link 1 and link 3

links is 0.5 .

Minimal-path set $P_{2}$ should be selected as the most important key path set by satisfying one of the following conditions:

1) Local government support for the links of $P_{2}$ is much greater than that for the links of $P_{1}$;

2) Local government support for links is relatively great.

The minimal-path set $P_{1}$ should be selected as the most important key path set when the above two conditions cannot be satisfied.

According to the abovementioned three cases of a sample bridge network, when minimal-path set $P_{1}$ is selected as the most important key path set, link 1 should be selected as the most important key link to improve traffic network reliability because $r_{10}<r_{20}$. When minimal-path set $P_{1}$ is selected as the most important key path set, link 4 should be selected as the most important key link to improve traffic network reliability because $r_{40}<r_{30}$.

\subsection{An Algorithm for Finding the Most Important Key Link in the Traffic Network}

In this section, an algorithm for finding the most important key link in any traffic network is proposed.

The basic procedure for finding the main minimal-path set and calculating the exact value of the terminal reliability of the minimal-path set was proposed by Wakabayashi. Thus, we start with the algorithm for the minimal-path set. The algorithm is shown as follows.

Step 1: Let $N$ be the number of minimal-path sets. The original reliability of all links in every minimal-path set is first stored in memory. For example, the original reliability of the links in the ith minimal-path set is stored in memory as two-dimensional array. Origin $[i][]$. Then, the original reliability of all the minimal-path sets is calculated and stored in memory as array Rops[]. Then, local government support for all links is stored in memory as array GSFL[].

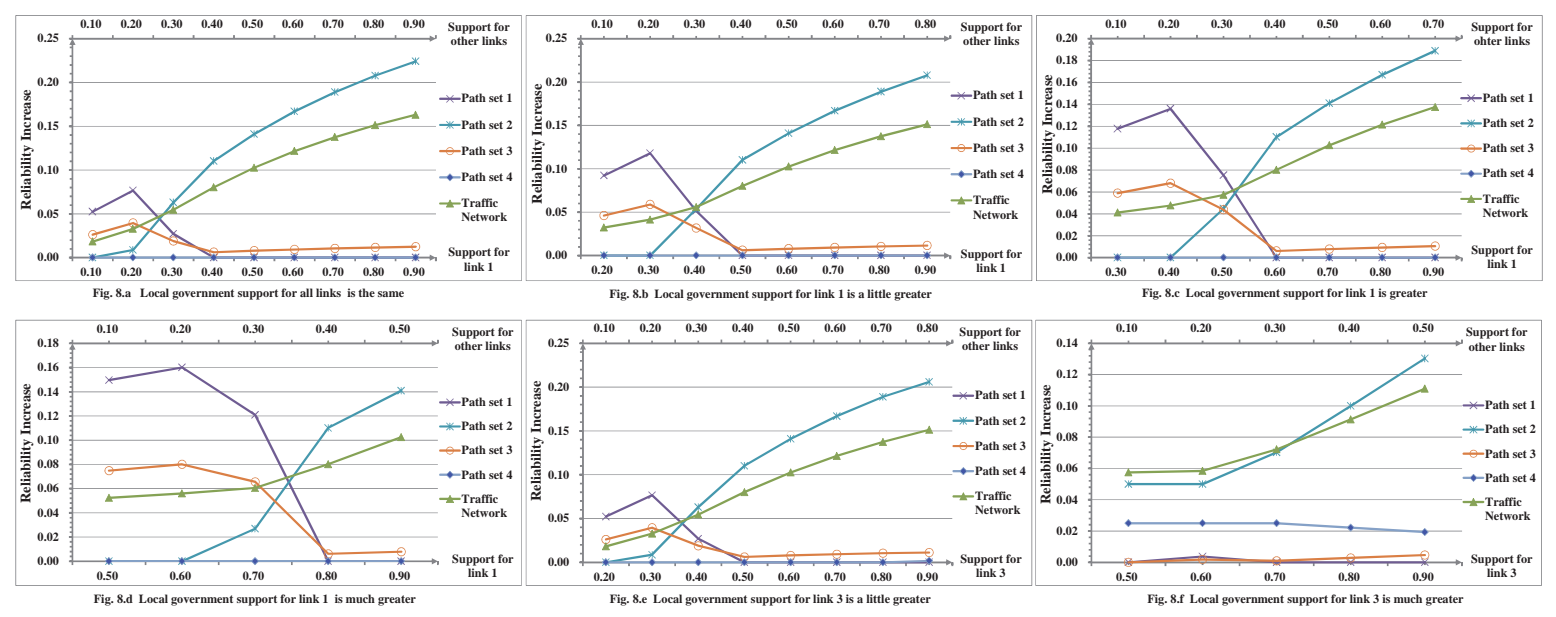

Fig. 8 Result for a very great difference in original link reliability between link 1 and link 3 
Step 2: The primary minimal-path sets are ascertained first. The least reliable primary minimal-path set and the most reliable primary minimal-path set are selected by reading array Rops[]. The least reliable primary minimal-path set is marked $L P$ and the most reliable primary minimal-path set is marked $H P$.

Step 3: The problem of finding the most important key minimal-path set is changed into the problem of finding the most important key link in a parallel network. $L P$ can be considered the least reliable link in a parallel network and $H P$ can be considered the most reliable link.

The critical point for local government support based on the value of LP and HP is calculated by using Eq. (23) and Eq. (24). Then, local government support for all links is judged to be relatively great or relatively small.

Step 4: Local government support for the links of $L P$ and HP is compared. If the difference in local government support between the group of links belonging to $L P$ and the group of links belonging to $H P$ is not great (in general, local government support for every link is not considerably different), then one proceeds to step 5, or else proceeds to step 6 .

Step 5: According to step 3, if local government support for all links is relatively small, $L P$ is the most important key minimal-path set and the least reliable link of $L P$ is the most important key link. If local government support for all links is relatively great, $H P$ is the most important key minimal-path set and the least reliable link of $H P$ is the most important key link.

After this judgment, one typically proceeds to step 7.

Step 6: This step is the special situation after a disaster. In general, this situation will not often happen.

When local government support for the links of $H P$ is greater than that for the links of $L P, H P$ is the most important key minimal-path set and the least reliable link of $H P$ is the most important key link.

When local government support for the links of $L P$ is greater than that for the links of $H P$, two selections are available:

If the original reliability of $L P$ is not much smaller than HP, LP is the most important key minimal-path set; or else one proceeds to step 5.

Step 7: End.

This algorithm is not suitable for all kinds of net- work, and so this is a potential unknown shortcoming.

\section{Conclusion}

In general, because of economic regional differences and limited resources, the central government cannot provide sufficient support for every disaster area after a mass disaster; local government support sometimes becomes more important for disaster prevention and reduction. In addition, because of the lack of material support after a disaster, post-disaster reconstruction may need to be sustained for somewhat longer; local government support is particularly important in this case.

In this paper, a method for improving traffic network reliability of roadways and highways was proposed based on local government support.

First, the current indices of traffic network reliability including RI, CI, and CIW were introduced and the shortcomings of these indices were identified.

Second, an improvement model of traffic network reliability based on local government support was proposed because of the circumstance in which only limited funds are available to reconstruct a damaged traffic system at the first stage of a disaster.

Third, to determine the model based on local government support for improving traffic network reliability, simulations of a parallel network and a simple bridge network were carried out based on limited capital for traffic restoration as defined in Eq. (24). From these simulations, the following conclusion is obtained.

When local government support for all links is relatively small, the least reliable link located in the least reliable primary minimal-path set should be selected as the most important key link to improve the traffic network. Conversely, when local government support for all links is relatively great, the least reliable link located in the most reliable primary minimal-path set should be selected as the most important key link to improve the traffic network.

Based on this model simulation, an algorithm for finding the most important key link in a general network was developed.

However, only two simple networks were simulated for determining the proposed model in this research. It is unclear whether the abovementioned conclusions can be obtained from larger and more 
complex networks. Furthermore, it is also unclear whether the abovementioned conclusions can be obtained when there is a change of entire limited capital for traffic restoration.

In future studies, larger and more complex networks should be simulated, the efficiency of traffic network reliability improvement when there is a change of entire limited capital for traffic restoration should be further discussed, and the algorithm for finding the most important key link in general networks should be further developed.

\section{REFERENCES}

Asakura, Y., Kashiwadani, M. and Fujiwara, K., 1998. Functional Hierarchy of a Road Network and Its Relations to Time Reliability, Journal of JSCE, No. 583/IV$38,51-60$.

Barlow, R.E. and Proschan, F., 1975. Statistical Theory of Reliability and Life Testing: Probability Models. Holt, Rinehart and Winston, New York, USA.

Birnbaum, Z.W., 1969. On the Importance of Different Components in a Multi-Component System. Multivariate Analysis II, Academic Press, New York, USA.

Fang, S.M. and Wakabayashi, H., 2010. Proceedings of the Fourth International Symposium on Transport Network Reliability. Minnesota University, USA.

Henley, E.J. and Kumamoto, H., 1981. Reliability Engineering and Risk Assessment. Prentice-Hall, Inc., Englewood Cliffs, USA.

Henley, E.J. and Kumamoto, H., 1992. Probabilistic Risk Assessment: Reliability Engineering, Design and Analysis. Institute of Electrical and Electronics Engineers, New York, USA.

Iida,Y. and Wakabayashi, H., 1988. An Efficient Calculation Method to Obtain Upper and Lower Bounds of Terminal Reliability of Road Networks using Boolean Algebra. Proceedings of JSCE, No. 395/IV-9, 75-84 (in Japanese).

Iida, Y. and Wakabayashi, H., 1989. An Approximation Method of Terminal Reliability of Road Networks using Partial Minimal Path and Cut Sets, Transport Policy, Management and Technology Towards 2001. Western Periodicals, Vol. 4, 2185-2198.

Lei, Q.X., Chen, W.F., Huang, D.F., Guo, H.M. and Wei, C.S., 2011. Decision-making Support System for Deployment of the Earthquake On-site Searching and Rescue Force. Journal of Seismological Research, Vol.
3, Yunnan, China.

Nicholson, A., 2007. Optimizing Network Terminal Reliability, Proceedings of the Third International Symposium on Transport Network Reliability. Delft University, Netherlands.

Wakabayashi, H. and Iida, Y., 1991. An Efficient Evaluation Method for Road Network Reliability in Disaster, International Symposium on Natural Disaster Reduction and Civil Engineering. JSCE, 397-405

Wakabayashi, H. and Iida, Y., 1992. Upper and Lower Bounds of Terminal Reliability of Road Networks: An Efficient Method with Boolean Algebra, Journal of Natural Disaster Science, 14(1), 29-44.

Wakabayashi, H. and Iida, Y., (1994). Improvement of Road Network Reliability with Traffic Management (Edited by Liu, B. and Blosseville, J.M.). Transportation Systems: Theory and Applications of Advanced Technology. Pergamum Elsevier Science, United Kingdom, pp. 603-608

Wakabayashi, H., 2004. Network Reliability Improvement: Probability Importance, Proceedings of Second International Symposium on Transport Network Reliability. University of Canterbury, Christchurch, New Zealand. 